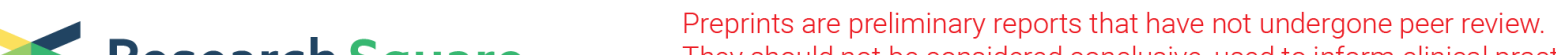 Research Square They should not be considered conclusive, used to inform clinical practice, or referenced by the media as validated information.
}

\section{TNF- $a$ activates WNT5A, which recruits osteoclast precursors by MCP-1 production, but not directly activates osteoclastogenesis, in psoriatic arthritis}

Shang-Hung Lin

Chang Gung Memorial Hospital Kaohsiung Branch https://orcid.org/0000-0002-7069-6993

Ji-Chen Ho

Chang Gung Memorial Hospital Kaohsiung Branch

Chung-Yuan Hsu

Chang Gung Memorial Hospital Kaohsiung Branch

Sung-Chou Li

Chang Gung Memorial Hospital Kaohsiung Branch

Wen-Yi Chou

Chang Gung Memorial Hospital Kaohsiung branch

Chang Chun Hsiao

Chang Gung Memorial Hospital Kaohsiung Branch

Chih-Hung Lee ( $\nabla$ dermlee@gmail.com)

Chang Gung Memorial Hospital Kaohsiung Branch

\section{Research Article}

Keywords: mdoc, wnt5a, osteoclasts, ligands, patients, osteoclastogenesis

Posted Date: November 1st, 2021

DOI: https://doi.org/10.21203/rs.3.rs-1016394/v1

License: (9) This work is licensed under a Creative Commons Attribution 4.0 International License.

Read Full License 


\section{Abstract \\ Background:}

Psoriatic arthritis (PsA) results from joint destruction by osteoclasts. Promising efficacy of TNF-a blockage indicates its important role in osteoclastogenesis of PsA. WNT ligands actively regulate osteoclastogenesis. We investigated how WNT ligands activate osteoclasts amid the TNF-a milieu in PsA.

\section{Methods:}

We first profiled the expression of WNT ligands in CD14+ monocyte-derived osteoclasts (MDOC) from 3 PsA patients and 3 healthy controls $(\mathrm{HC})$ and then validated the candidate WNT ligands in 32 PsA patients and $16 \mathrm{HC}$. Through RNA interference against WNT ligands in MDOC, we determined the mechanisms by which TNF-a exerts its effects on osteclastogenesis or chemotaxis.

\section{Results:}

The results showed numbers of CD68+WNT5A+ osteoclasts are increased in PsA joints. WNT5A was selectively upregulated by TNF-a in MDOC from PsA patients. However, direct osteoclastogenesis effect (RANK expression) by TNF-awas not inhibited by WNT5A siRNA. Instead, CXCL1, CXCL16, and MCP-1 was selectively increased in supernatants of MDOC from PsA patients. RNA interference against WNT5A abolished the increased MCP-1 from MDOC and THP-1-cell-derived osteoclasts. The increased migration of osteoclast precursors (OCP) induced by supernatant from PSA MDOC was abolished by MCP-1 neutralizing antibody. WNT5A and MCP-1 expressions were decreased in MDOC from PsA patients treated by biologics against TNF-a but not IL-17.

\section{Conclusion:}

We conclude TNF-a recruits OCP by WNT5A-mediated MCP-1 production but not directly activates osteoclastogenesis in PsA.

\section{Introduction}

Psoriatic arthritis (PSA) is a chronic inflammatory joint disease. Unrecognized joint damage can lead to permanent joint deformity and functional impairment [1-3]. The joint destruction is associated with multifocal bony erosion and resorption by active osteoclasts. Tissue osteoclasts are derived from precursors of the monocyte/macrophage lineage [4-6]. The differentiation of the osteoclasts from their monocyte precursors is tightly regulated by a cascade of integrated signaling steps, including the macrophage colony stimulating factor (M-CSF) and the receptor activator of NF-kB ligand (RANKL) [6]. M- 
CSF induces the differentiation of osteoclast precursors (OCP) from monocyte precursors, along with the induction of receptor activator of nuclear factor-KB (RANK) on the cell surface [7]. In PsA, RANKL is upregulated in the synovial lining of affected synovial sheaths of tendons. The binding of RANKL to RANK on OCP derived from circulating CD14+ monocytes triggers the proliferation of OCP and their differentiation into the multinucleated osteoclasts [8]. Ritchlin et al. showed a marked increase in circulatory OCP in PsA patients compared to healthy controls (HC). Tumor necrosis factor-a (TNF-a), one of the major cytokines involved in the pathogenesis of bony resorption in PsA, increases the number of circulating OCP [8]. The level of monocyte chemoattractant protein-1 (MCP-1), a major chemotactic protein that recruits C-C chemokine receptor type 2 (CCR-2)-expressing circulatory OCP to tissue, [9- 11] is significantly increased in the synovial fluid in PsA patients [12]. In addition, localized MCP-1 expression in PsA synovium recruits not only OCP but also T cells [13].

Wingless-type MMTV integration site family member (Wnt) signaling is important in balancing bone formation and bone resorption through the regulation of osteoclast differentiation from OCP [14]. Among the 19 Wnt ligands, Wnt3a, Wnt4, Wnt5a, and Wnt16 have been documented to regulate osteoclast differentiation from OCP [14], and Wnt5a also enhances the expression of RANK on OCP [15]. An autocrine loop of Wnt ligands in the osteoclasts and OCP augments the differentiation of OCP into osteoclasts [16-18].

In rheumatoid arthritis, WNT5A has been found to be upregulated in the synovium, along with enhanced production of IL-6 and IL-15 [19]. However, the WNT signaling profiles in CD14+ monocyte-derived osteoclasts (MDOC) from PsA patients have never been investigated. This study aimed to unravel the regulatory mechanisms involving WNT signaling in osteoclast activation in PsA.

\section{Materials And Methods}

Isolation of human circulatory CD14+ monocytes to profile WNT ligands in patients with PsA and HC

The clinical manifestations of PsA include peripheral arthritis, axial arthritis, enthesitis or dactylitis, and skin and/or nail involvement. In the PsA group in the present study, all the patients fulfilled the Classification for Psoriatic Arthritis (CASPAR) criteria for the diagnosis of PsA. The cases of PsA were confirmed by both dermatologists and rheumatologists. The $\mathrm{HC}$ were examined thoroughly to ensure the absence of any psoriatic lesions or inflammatory joint pain. Patients who had active infections were excluded. All the patients provided written informed consent approved by the IRB of Chang Gung Memorial Hospital (IRB-201802336A3). Patient blood samples were processed to remove red blood cells, followed by the collection of buffy coats enriched with peripheral blood mononuclear cells (PBMCs). Circulating CD14+ monocytes were isolated from PBMCs using CD14+ MicroBeads (Miltenyi Biotec, Germany). The purity of the CD14+ cells after selection was approximately $96.4 \%$ according to flow cytometry analysis based on our previous study [20]. Quantitative real-time polymerase chain reaction (qRT-PCR) was performed on the Roche LightCycler ${ }^{\circledR} 96$ System (Roche Applied Science, Upper Bavaria, Germany) with the Fast SYBR Green PCR Master Mix (Applied Biosystems; Thermo Fisher Scientific, Inc, 
Carlsbad, CA, USA) using samples of RNA from the CD14+ monocytes of $\mathrm{HC}$ and patients with PsA. The PCR program consisted of an initial denaturation at $95^{\circ} \mathrm{C}$ for 20 seconds followed by 45 cycles of qRTPCR at $95^{\circ} \mathrm{C}$ for 3 seconds (denaturation) and $60^{\circ} \mathrm{C}$ for 30 seconds (annealing, extension, and reading fluorescence). The primer sequences for the different WNT ligands are listed in Supplementary Table 1.

\section{Differentiation of osteoclasts (MDOC) from human circulatory CD14+ monocytes}

Purified human CD14+ monocytes were seeded at $2.5 \times 10^{5}$ cells/well in 96-well plates containing aminimum essential medium (a-MEM) with fetal bovine serum (FBS) $(10 \%, v / v$; Invitrogen, Waltham, MA) and M-CSF (20 ng/mL; PeproTech, Rocky Hill, NJ) for 3 days. RANKL $(25 \mathrm{ng} / \mathrm{mL})$ and TNF-a $(50 \mathrm{ng} / \mathrm{mL})$ (both from PeproTech, Rocky Hill, NJ, USA) were added every 3 days for 9 days to induce osteoclast differentiation. The osteoclasts were identified by staining with tartrate-resistant acid phosphatase (TRAP) on Day 13 using the Acid Phosphate Leukocyte Kit (Sigma, St. Louis, MO). TRAP-stained cells containing three or more nuclei were defined as osteoclasts. [21] The numbers of osteoclasts were counted and averaged from four high-power fields (HPFs) (100x) per well.

\section{Differentiation of osteoclasts from THP-1 cells}

THP-1 cells (Bioresource Collection and Research Center, Taiwan) were seeded at $3 \times 10^{5}$ cells/well in 24well plates containing RPMI 1640 with FBS (10\%, v/v; Invitrogen, Waltham, MA) and PMA (160 nM; Sigma-Aldrich, St Louis, MO) for 1 day. M-CSF $(20 \mathrm{ng} / \mathrm{mL}$ ) was then added on Day 2, and RANKL (25 $\mathrm{ng} / \mathrm{mL}$; PeproTech, Rocky Hill, NJ) and TNF-a (25 ng/mL; PeproTech, Rocky Hill, NJ) were added on Days 2 and 5 to induce osteoclast differentiation. The osteoclasts were stained with TRAP on Day 8.

The details about western blot analysis, immunohistochemistry, RNA interference, Human cytokine and chemokine antibody array, cell migration, flow cytometry and statistical analysis are provided in the Supplementary Materials and Methods.

\section{Results}

\section{The demographics of patients with PsA and HC}

Thirty-two patients with PsA (male/female: 16/16; average age: 48.3 years old) and $16 \mathrm{HC}$ (male/female: 8/8; average age: 46.4 years old) were identified. All the PsA patients had peripheral arthritis, including $31.3 \%$ with axial arthritis, $40.6 \%$ with dactylitis, and $62.5 \%$ with enthesitis (supplementary Table 2).

Increased transcription and translation of WNT5A in MDOC and tissue osteoclasts from affected joints in PsA patients

We conducted a small pilot study to profile the transcriptional levels of all the WNT ligands using qRTPCR in CD14+ monocytes and MDOC from PsA patients $(n=3)$ and $H C(n=3)$. Among the WNT ligands, the 
expression level of WNT5A was selectively increased 15-fold in MDOC from PsA patients compared to the level in MDOC from the $\mathrm{HC}(\mathrm{p}<0.05)$ (Figure 1a). To validate the selective upregulation of WNT5A in MDOC from PsA patients, we measured the RNA expression level of WNT5A in MDOC from more PsA patients $(n=32)$ and $\mathrm{HC}(\mathrm{n}=16)$. The results showed that the transcriptional expression of WNT5A was higher in the MDOC from PsA patients than in those from the $\mathrm{HC}(p=0.0001)$ (Figure 1b). As we had observed an increase in the expression of WNT5A mRNA in the MDOC from PsA patients, we next examined whether the level of the WNT5A protein was similarly increased using Western blotting. The results showed that the level of the WNT5A protein was higher in MDOC from PsA patients $(n=3)$ than in those from HC $(n=3)(p<0.05)$ (Figure 1c). Furthermore, we investigated whether WNT5A expression was increased in osteoclasts in the destructive joints of PsA patients. We collected joint tissues from PsA patients $(n=5)$ and osteoarthritic patients $(n=5)$ who had received joint replacement and stained them with WNT5A and CD68 using immunohistochemical staining. The results showed increased numbers of WNT5A- and CD68-expressing osteoclasts in the joints of the PsA patients compared to those of the osteoarthritis patients $(p=0.0019)$ (Figure $1 d)$. We confirmed that the expression of WNT5A was selectively increased in the osteoclasts of PsA patients.

\section{TNF- $a$ activates WNT5A pathway, which is independent of osteoclastogenesis in PsA}

WNT5A was highly expressed in the MDOC of the PsA patients. We investigated which cytokines contributed to this increased expression level. RNA samples from monocytes and MDOC were analyzed using qRT-PCR. The results show that the expression of WNT5A was significantly increased in MDOC by M-CSF, RANKL and TNF-a treatment compared to that with medium only and/or M-CSF+ RANKL treatment $(p<0.05)$ (Figure 2a). These results indicate that TNF-a significantly increased the expression of WNT5A in MDOC from PsA patients. The previous study showed that Wnt5a increased the expression of Rank and active osteoclastogenesis in a murine arthritis model. [15] We then investigated whether RANK mediated osteoclastogenesis in PsA. As anticipated, the expression level of RANK was higher in MDOC following RANKL and RANKL+ TNF-a treatment than following treatment with medium only $(\mathrm{p}<0.05)$ (Figure 2a). We also explored whether WNT5A regulated RANK or osteoclastogenesis. After WNT5A interference, we measured the expression levels of WNT5A and RANK using qRT-PCR and the number of osteoclasts using TRAP staining. The results showed that WNT5A mRNA was significantly downregulated in the WNT5A siRNA but not in the control siRNA group $(p<0.05)$ (Figure $2 b)$. The number of TRAP+ osteoclasts was induced after combined M-CSF, RANKL, and TNF-a treatment, it was not changed by WNT5A RNA interference (Figure 2c and d). The increased expression level of RANK after combined M-CSF, RANKL, and TNF-a treatment was not inhibited by WNT5A siRNA (Figure 2e). These results suggest that WNT5A does not directly contribute to active osteoclastogenesis and RANK expression in MDOC from PsA patients.

\section{Selective induction of MCP-1, but not CXCL1 or CXCL16, by WNT5A in MDOC from PsA patients}


We wanted to determine whether chemokine or cytokine production was increased in the MDOC of the PsA patients compared to those of the HC. The supernatants from the MDOC of the PsA ( $n=3)$ patients and $\mathrm{HC}(\mathrm{n}=3)$ were analyzed using a multiplex chemokine assay. Among 36 chemokines, the results showed higher expression levels of CXCL1, CXCL16, and MCP-1 in the supernatants of the MDOC from the PsA patients than those from the $\mathrm{HC}(\mathrm{p}<0.05)$ (Figure $3 a$ and $b)$. We further investigated whether WNT5A regulated the production of CXCL1, CXCL16, and MCP-1. The levels of the three cytokines in the supernatants of monocytes and MDOC with/without WNT5A RNA interference were measured using ELISA. The results show that the production of CXCL1, CXCL16 and MCP-1, was increased in MDOC from PsA patients and that the enhanced MCP-1 production (but not that of CXCL1 or CXCL16) in the MDOC from the PsA patients was significantly decreased by WNT5A blockade (Figure 3c, $d$ and e). WNT5A regulated the production of MCP-1 in MDOC from PsA patients.

\section{TNF- $a$ increased MCP-1 production through WNT5A in THP- 1-cell-derived osteoclasts}

We found increased expression of WNT5A in MDOC in patients with PSA. We next investigated whether the upregulation of WNT5A by TNF-a could be recapitulated in vitro and sought to decipher its mechanism. We differentiated THP-1 cells into osteoclasts to investigate whether WNT5A increased after TNF-a treatment in osteoclasts. RNA samples from THP-1-derived osteoclasts were analyzed using qRTPCR. The results showed that combined M-CSF, RANKL, and TNF- $a$ treatment induced WNT5A expression, which was significantly reduced in cells transfected with WNT5A siRNA (Figure 4a). In parallel, we investigated whether WNT5A interference abrogated the expression of RANK. The expression level of RANK increased after M-CSF, RANKL and TNF-a treatment, it is not decreased by WNT5A RNA interference. (Figure $4 \mathrm{~b}$ ). We then explored whether WNT5A interference abrogated the production of CXCL1, CXCL16, and MCP-1. The concentrations of CXCL1 for THP-1 cells with medium only, THP-1 cells with PMA treatment, THP-1-cell-derived osteoclasts, THP-1-cell-derived osteoclasts with control siRNA, and THP-1-cell-derived osteoclasts with WNT5A siRNA $(100 \mu \mathrm{g} / \mathrm{mL})$ were low and did not significantly differ among the groups (Figure 4c). Although the production of CXCL16 was induced after combined MCSF, RANKL, and TNF-a treatment, it was not changed by WNT5A RNA interference (Figure 4d). Notably, the production of MCP-1 was induced after the combined M-CSF, RANKL, and TNF-a treatment but was decreased by more than $50 \%$ by WNT5A RNA interference (Figure 4e).

\section{MCP-1 in supernatants of MDOC helps to recruit OCP in PsA}

We observed increased MCP-1 in the supernatant of MDOC from PsA patients; we then explored whether this recruited more OCP. The supernatants of MDOC from PsA patients $(n=5)$ were treated with MCP-1 antibody or a mock antibody. We tested the direct effects of supernatant from MDOC with/without MCP-1 antibody on monocyte migration using Transwell assays in which CD14+ monocytes from $\mathrm{HC}$ were added to the upper chamber and assessed for their response to chemotactic stimuli from the culture supernatant of MDOC from PsA patients, which was added to the lower chamber. The ratio of CD14+ monocyte migration was increased when the supernatants were added, and the amount of migration was similar to that induced by the addition of MCP-1 at $1500 \mathrm{pg} / \mathrm{mL}$ (Figure $5 \mathrm{a}$ ). MCP-1 antibody treatment at 
20 or $40 \mu \mathrm{g} / \mathrm{mL}$ significantly reduced the enhancement of the migration of MDOC by the supernatant. Furthermore, we wanted to determine whether MCP-1 in the supernatant of MDOC could recruit more CCR2+RANK-expressing OCP. Coupled with a chemotactic assay and flow cytometry to identify OCP, the data show that the percentages of CCR2+RANK-expressing OCP in peripheral CD14+ monocytes, the upper chamber and the lower chamber were $1.5 \pm 0.5 \%, 2 \pm 1.2 \%$, and $1.5 \pm 1.1 \%$, respectively (Figure $5 b$ ). The results indicate that the increased MCP-1 in the supernatants of the MDOC from PsA patients recruited high numbers of CCR2+RANK-expressing OCP.

\section{Both WNT5A expression and MCP-1 production in MDOC of PsA patients were decreased by TNF-a blockade}

We observed that WNT5A-mediated MCP-1 production in MDOC recruits OCP in PsA. We next investigated whether TNF-a or IL-17 modulated WNT5A expression in MDOC in PsA. For this, MDOC were cultivated from PsA patients as described above. On Days 3 and 9, cells were treated with different concentrations of TNF-a or IL-17 inhibitors. WNT5A was upregulated as expected when CD14+ cells were treated with combined M-CSF, RANKL, and TNF-a. The upregulation of WNT5A was abolished by TNF-a blockers including etanercept and adalimumab, but not the IL-17 blocker secukinumab (Figure 6a). We were also interested in whether the production of MCP-1 from MDOC was mediated by TNF- $a$ or IL-17. The concentrations of CXCL1, CXCL16, and MCP-1 in the supernatants of MDOC treated with or without TNFa blockers or IL-17 blockers were measured using ELISA. The results show that productions of CXCL1 and CXCL16 increased after M-CSF, RANKL, and TNF- $a$ treatment, they were not changed by anti-TNF- $a$ treatment (etanercept, 400 or $800 \mu \mathrm{g} / \mathrm{mL}$, or adalimumab, 400 or $800 \mu \mathrm{g} / \mathrm{mL}$ ) or anti-IL-17A treatment (secukinumab, 400 and $800 \mu \mathrm{g} / \mathrm{mL}$ ) (Figure $6 \mathrm{~b}$ and c). However, the production of MCP-1 was enhanced in cells that received combined M-CSF, RANKL, and TNF-a treatments. The enhancement was reduced by more than $50 \%$ when cells were treated with TNF-a blockers including etanercept and adalimumab, but not with the IL-17A blocker secukinumab (Figure 6d). Overall, we provide compelling evidence that TNF-a mediates WNT5A-dependent MCP-1 production, which drives the migration of OCP to the PsA joint.

\section{Discussion}

This is the first study to investigate the role of WNT signaling in MDOC from patients with PsA. Our results reveal higher WNT5A expression levels in MDOC from PsA patients than in those from HC. In addition, WNT5A expression was increased in osteoclasts in the damaged PsA joints compared to that in those from osteoarthritis. MCP-1-mediated OCP migration could be abolished by WNT5A RNA interference and blocking TNF-a (but not by blocking IL-17).

The WNT signaling pathways are involved in physiological bone metabolism [14]. Our results show higher expression levels of WNT5A mRNA and protein in MDOC from PsA patients than in those from HC. The increased expression of WNT5A in the osteoclasts of destructive joints confirms its pathogenic role in active osteoclastogenesis in PsA patients. Previous studies showed that TNF-a induced the expression 
of WNT5A in human monocytes [21] and dental pulp cells [22]. WNT5A expression was increased in the synovial fluids of patients with spondyloarthropathy compared to those with osteoarthritis [23]. Our results consistently show increased expression of WNT5A after TNF-a treatment in the MDOC of PsA patients. Wnt5a promotes osteoclast differentiation and function via RANK expression, thereby enhancing RANKL-induced osteoclastogenesis in mouse models [15]. The knockout of late-stage osteoclast-specific Ror2, a protein downstream of Wnt5a, increases bone mass [24, 25]. However, our results show that WNT5A interference did not decrease the expression of RANK and inhibit osteoclastogenesis. Instead, WNT5A regulates MCP-1 production, which recruits OCP. In fact, WNT5A has been reported to upregulate MCP-1 expression in macrophages [26]. The WNT5A treatment of human dental pulp cells increased the production of cytokines and chemokines, including IL-8, CXCL1, MCP-1, and CCL5 [22]. This result may reflect the chronic inflammatory burden in PsA.

Increased production of MCP-1, RANTES, CXCL1, CXCL8/IL8, and CXCL9, as well as CCR2 and CCR5, has been described in both the synovial tissue and the synovial fluid of patients with PsA $[10,13,27]$. Increased MCP-1 in the serum was found to be a potential biomarker for distinguishing PsA from osteoarthritis [28]. Our results show increased concentrations of MCP-1, CXCL1, and CXCL16 in the supernatants of MDOC from PSA patients. Furthermore, the results confirm that WNT5A interference could selectively inhibit the production of MCP-1 from MDOC in PSA patients. In addition, the results confirm that the production of MCP-1 by THP-1-cell-derived osteoclasts was regulated by WNT5A. Researchers have shown that increased CXCL16 contributed to the retention of CXCR6+ Tc17 cells in PsA synovial fluid [29]. CXCL16 could be produced as a transmembrane-bound chemokine by monocytes, macrophages, and dendritic cells; CXCL16 may contribute to their recruitment and persistence in the inflamed PsA joint [29]. Cytological analysis revealed CXCL1 mRNA to be located mainly in monocytic cells in the synovial fluid of PsA patients [27]. Hardaway et al. reported that CXCL1 could stimulate osteoclast differentiation in vitro [30].

MCP-1 was induced during TNF-a-mediated osteoclast differentiation. [31] Early work showed that the recruitment of monocytes to the bone surface is mediated by MCP-1 [32, 33]. The link between mechanical strain and the onset of arthritis appears to depend on the local recruitment of Ly $6^{\text {high }}$ inflammatory monocytes elicited by the mechanostress-induced MCP-1/CCR2 axis [34]. MCP-1 was reported to serve as a chemotactic signal for OCP via the CCR2 receptor [35]. Our results indicate that MCP-1 in the supernatants of MDOC from PsA patients recruits CCR2+RANK-expressing OCP. The number of OCP was decreased in PsA patients by successful anti-TNF-a treatment [8]. The good clinical responses to TNF-a inhibition in patients with PsA are well known, although the mechanisms could be multifactorial [36]. One previous study showed that the high numbers of OCP in the peripheral blood of PsA patients were decreased significantly by anti-TNF-a agents [37]. However, the impact of OCP regulation is not known. Anti-TNF-a treatment has been reported to decrease MCP-1 production from cultured mononuclear cells from the synovial fluid of patients with PsA [38]. Our results show that antiTNF-a agents (etanercept and adalimumab) decreased both WNT5A expression and MCP-1 production. 
Furthermore, the decreased recruitment of OCP through WNT5A represents one plausible mechanism independent of blocking osteoclastogenesis for the clinical efficacy of TNF- $a$ inhibitors in treating PsA.

\section{Conclusions}

This study revealed increased WNT5A expression in MDOC from PsA patients. TNF-a increases MCP-1 production from MDOC through WNT5A upregulation in patients with PSA. Anti-TNF-a agents decreased OCP recruitment through MCP-1 inhibition.

\section{Declarations}

\section{Ethics approval and consent to participate}

This study was approved by the Institutional Review Board of Chang Gung Memorial Hospital (IRB201802336A3). All the individual data were anonymous before analysis, and informed consent was waived.

\section{Consent for publication}

Not applicable.

\section{Availability of supporting data and materials}

The authors confirm that the data supporting the findings of the present study are available within the manuscript and the supplemental data.

\section{Competing interests}

The authors have declared that no competing interests exist.

\section{Funding}

The authors received grants from Ministry of Science and Technology of Taiwan (MOST 108-2314-B182A-105 -MY3) and the Chang-Gung Memorial Foundation (CMRPG8J0411). The funders had no role in the study design, data collection and analysis, decision to publish, or preparation of the manuscript.

\section{Authors' contributions}

SHL designed the study, conducted the experiments, analyzed the

results and wrote the manuscript. $\mathrm{CHL}$ and $\mathrm{CCH}$ contributed to study design, analyzed the results and critically revised the paper. $\mathrm{SCL}$ contributed to data analysis. $\mathrm{JCH}, \mathrm{CYH}$, and WYC contributed to data acquisition. 


\section{Acknowledgments}

We thank the Genomics \& Proteomics Core Laboratory, Department of Medical Research, Kaohsiung Chang Gung Memorial Hospital for technical support.

\section{Authors' details}

${ }^{1}$ Department of Dermatology, Kaohsiung Chang Gung Memorial Hospital and Chang Gung University College of Medicine, Kaohsiung. ${ }^{2}$ Graduate Institute of Clinical Medical Sciences, College of Medicine, Chang Gung University, Taiwan. ${ }^{3}$ Division of Rheumatology, Allergy, and Immunology, Department of Internal Medicine, Kaohsiung Chang Gung Memorial Hospital and Chang Gung University College of Medicine, Kaohsiung, Taiwan. ${ }^{4}$ Genomics and Proteomics Core Laboratory, Kaohsiung Chang Gung Memorial Hospital, Chang Gung University College of Medicine, Kaohsiung, Taiwan. ${ }^{5}$ Department of Orthopaedic Surgery, Kaohsiung Chang Gung Memorial Hospital and Chang Gung University College of Medicine, Kaohsiung City, Taiwan. ${ }^{6}$ Division of Pulmonary and Critical Care Medicine, Kaohsiung Chang Gung Memorial Hospital and Chang Gung University College of Medicine, Kaohsiung City, Taiwan.

\section{References}

1. Ritchlin CT, Colbert RA, Gladman DD: Psoriatic Arthritis. The New England journal of medicine 2017, 376(10):957-970.

2. Veale DJ, Fearon U: The pathogenesis of psoriatic arthritis. Lancet 2018, 391(10136):2273-2284.

3. Anandarajah AP, Ritchlin CT: The diagnosis and treatment of early psoriatic arthritis. Nature reviews Rheumatology 2009, 5(11):634-641.

4. Teitelbaum SL: Bone resorption by osteoclasts. Science 2000, 289(5484):1504-1508.

5. Massey HM, Flanagan AM: Human osteoclasts derive from CD14-positive monocytes. British journal of haematology 1999, 106(1):167-170.

6. Nakashima T, Takayanagi H: Osteoimmunology: crosstalk between the immune and bone systems. $J$ Clin Immunol 2009, 29(5):555-567.

7. Chiu YH, Mensah KA, Schwarz EM, Ju Y, Takahata M, Feng C, McMahon LA, Hicks DG, Panepento B, Keng PC et al: Regulation of human osteoclast development by dendritic cell-specific transmembrane protein (DC-STAMP). J Bone Miner Res 2012, 27(1):79-92.

8. Ritchlin CT, Haas-Smith SA, Li P, Hicks DG, Schwarz EM: Mechanisms of TNF-alpha- and RANKLmediated osteoclastogenesis and bone resorption in psoriatic arthritis. The Journal of clinical investigation 2003, 111(6):821-831.

9. Geissmann F, Jung S, Littman DR: Blood monocytes consist of two principal subsets with distinct migratory properties. Immunity 2003, 19(1):71-82. 
10. Komano Y, Nanki T, Hayashida K, Taniguchi K, Miyasaka N: Identification of a human peripheral blood monocyte subset that differentiates into osteoclasts. Arthritis research \& therapy 2006, 8(5):R152.

11. Matsubara R, Kukita T, Ichigi Y, Takigawa I, Qu PF, Funakubo N, Miyamoto H, Nonaka K, Kukita A: Characterization and identification of subpopulations of mononuclear preosteoclasts induced by TNF-alpha in combination with TGF-beta in rats. PloS one 2012, 7(10):e47930.

12. Haringman JJ, Ludikhuize J, Tak PP: Chemokines in joint disease: the key to inflammation? Annals of the rheumatic diseases 2004, 63(10):1186-1194.

13. Ross EL, D'Cruz D, Morrow WJ: Localized monocyte chemotactic protein-1 production correlates with T cell infiltration of synovium in patients with psoriatic arthritis. The Journal of rheumatology 2000 , 27(10):2432-2443.

14. Lerner UH, Ohlsson C: The WNT system: background and its role in bone. J Intern Med 2015, 277(6):630-649.

15. Maeda K, Kobayashi Y, Udagawa N, Uehara S, Ishihara A, Mizoguchi T, Kikuchi Y, Takada I, Kato S, Kani S et al: Wnt5a-Ror2 signaling between osteoblast-lineage cells and osteoclast precursors enhances osteoclastogenesis. Nature medicine 2012, 18(3):405-412.

16. Pederson L, Ruan M, Westendorf JJ, Khosla S, Oursler MJ: Regulation of bone formation by osteoclasts involves Wnt/BMP signaling and the chemokine sphingosine-1-phosphate. Proceedings of the National Academy of Sciences of the United States of America 2008, 105(52):20764-20769.

17. Wei W, Zeve D, Suh JM, Wang X, Du Y, Zerwekh JE, Dechow PC, Graff JM, Wan Y: Biphasic and dosage-dependent regulation of osteoclastogenesis by beta-catenin. Mol Cell Biol 2011, 31(23):4706-4719.

18. Santiago F, Oguma J, Brown AM, Laurence J: Noncanonical Wnt signaling promotes osteoclast differentiation and is facilitated by the human immunodeficiency virus protease inhibitor ritonavir. Biochemical and biophysical research communications 2012, 417(1):223-230.

19. Sen M, Lauterbach K, El-Gabalawy H, Firestein GS, Corr M, Carson DA: Expression and function of wingless and frizzled homologs in rheumatoid arthritis. Proceedings of the National Academy of Sciences of the United States of America 2000, 97(6):2791-2796.

20. Lin SH, Chuang HY, Ho JC, Lee CH, Hsiao CC: Treatment with TNF-alpha inhibitor rectifies M1 macrophage polarization from blood CD14+ monocytes in patients with psoriasis independent of STAT1 and IRF-1 activation. Journal of dermatological science 2018.

21. Li X, Wang J, Zhan Z, Li S, Zheng Z, Wang T, Zhang K, Pan H, Li Z, Zhang N et al: Inflammation Intensity-Dependent Expression of Osteoinductive Wnt Proteins Is Critical for Ectopic New Bone Formation in Ankylosing Spondylitis. Arthritis Rheumato/ 2018, 70(7):1056-1070.

22. Zhao Y, Wang CL, Li RM, Hui TQ, Su YY, Yuan Q, Zhou XD, Ye L: Wnt5a promotes inflammatory responses via nuclear factor kappaB (NF-kappaB) and mitogen-activated protein kinase (MAPK) pathways in human dental pulp cells. J Biol Chem 2014, 289(30):21028-21039. 
23. Bougault C, Briolay A, Boutet MA, Pilet P, Delplace S, Le Goff B, Guicheux J, Blanchard F, Magne D: Wnt5a is expressed in spondyloarthritis and exerts opposite effects on enthesis and bone in murine organ and cell cultures. Trans/ Res 2015, 166(6):627-638.

24. Uehara S, Udagawa N, Mukai H, Ishihara A, Maeda K, Yamashita T, Murakami K, Nishita M, Nakamura T, Kato $S$ et al: Protein kinase N3 promotes bone resorption by osteoclasts in response to Wnt5a-Ror2 signaling. Sci Signa/2017, 10(494).

25. Uehara S, Udagawa N, Kobayashi Y: Non-canonical Wnt signals regulate cytoskeletal remodeling in osteoclasts. Cellular and molecular life sciences: CMLS 2018, 75(20):3683-3692.

26. Zhao C, Bu X, Wang W, Ma T, Ma H: GEC-derived SFRP5 inhibits Wnt5a-induced macrophage chemotaxis and activation. PloS one 2014, 9(1):e85058.

27. Konig A, Krenn V, Toksoy A, Gerhard N, Gillitzer R: Mig, GRO alpha and RANTES messenger RNA expression in lining layer, infiltrates and different leucocyte populations of synovial tissue from patients with rheumatoid arthritis, psoriatic arthritis and osteoarthritis. Virchows Arch 2000, 436(5):449-458.

28. Chandran V, Abji F, Perruccio AV, Gandhi R, Li S, Cook RJ, Gladman DD: Serum-based soluble markers differentiate psoriatic arthritis from osteoarthritis. Annals of the rheumatic diseases 2019, 78(6):796-801.

29. Steel KJA, Srenathan U, Ridley M, Durham LE, Wu SY, Ryan SE, Hughes CD, Chan E, Kirkham BW, Taams LS: Polyfunctional, Proinflammatory, Tissue-Resident Memory Phenotype and Function of Synovial Interleukin-17A+CD8+ T Cells in Psoriatic Arthritis. Arthritis Rheumatol 2020, 72(3):435447.

30. Hardaway AL, Herroon MK, Rajagurubandara E, Podgorski I: Marrow adipocyte-derived CXCL1 and CXCL2 contribute to osteolysis in metastatic prostate cancer. Clin Exp Metastasis 2015, 32(4):353368.

31. Hounoki H, Sugiyama E, Mohamed SG, Shinoda K, Taki H, Abdel-Aziz HO, Maruyama M, Kobayashi $M$, Miyahara T: Activation of peroxisome proliferator-activated receptor gamma inhibits TNF-alphamediated osteoclast differentiation in human peripheral monocytes in part via suppression of monocyte chemoattractant protein-1 expression. Bone 2008, 42(4):765-774.

32. Rahimi P, Wang CY, Stashenko P, Lee SK, Lorenzo JA, Graves DT: Monocyte chemoattractant protein1 expression and monocyte recruitment in osseous inflammation in the mouse. Endocrinology 1995, 136(6):2752-2759.

33. Deshmane SL, Kremlev S, Amini S, Sawaya BE: Monocyte chemoattractant protein-1 (MCP-1): an overview. J Interferon Cytokine Res 2009, 29(6):313-326.

34. Cambre I, Gaublomme D, Burssens A, Jacques P, Schryvers N, De Muynck A, Meuris L, Lambrecht S, Carter S, de Bleser $\mathrm{P}$ et al: Mechanical strain determines the site-specific localization of inflammation and tissue damage in arthritis. Nature communications 2018, 9(1):4613.

35. Sumi K, Abe T, Kunimatsu R, Oki N, Tsuka Y, Awada T, Nakajima K, Ando K, Tanimoto K: The effect of mesenchymal stem cells on chemotaxis of osteoclast precursor cells. J Oral Sci 2018, 60(2):221- 
225.

36. Schett G, Elewaut D, Mclnnes IB, Dayer JM, Neurath MF: How cytokine networks fuel inflammation: Toward a cytokine-based disease taxonomy. Nature medicine 2013, 19(7):822-824.

37. Anandarajah AP, Schwarz EM, Totterman S, Monu J, Feng CY, Shao T, Haas-Smith SA, Ritchlin CT:

The effect of etanercept on osteoclast precursor frequency and enhancing bone marrow oedema in patients with psoriatic arthritis. Annals of the rheumatic diseases 2008, 67(3):296-301.

38. Nielsen MA, Lomholt S, Mellemkjaer A, Andersen MN, Buckley CD, Kragstrup TW: Responses to Cytokine Inhibitors Associated with Cellular Composition in Models of Immune-Mediated Inflammatory Arthritis. ACR Open Rheumatol 2020, 2(1):3-10.

\section{Figures}

a

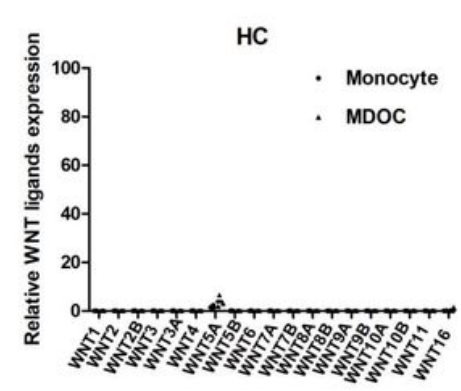

C

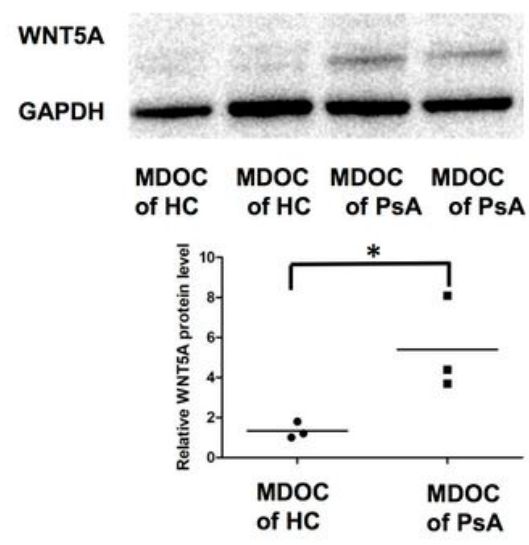

b

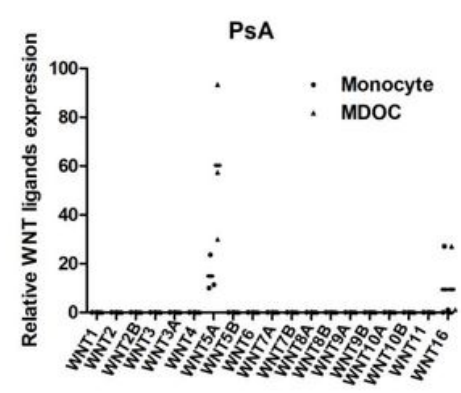

d

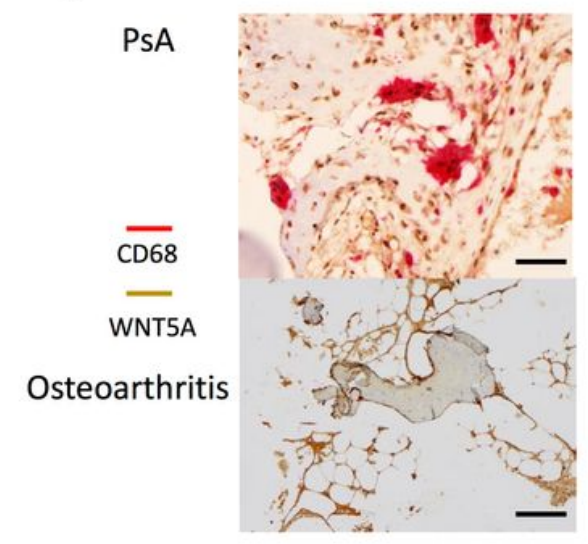

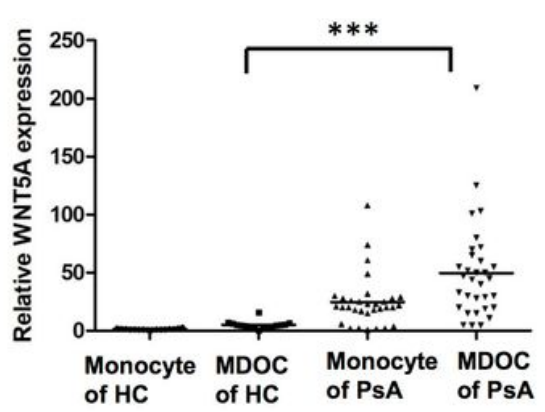

of $\mathrm{HC}$ of $\mathrm{HC}$

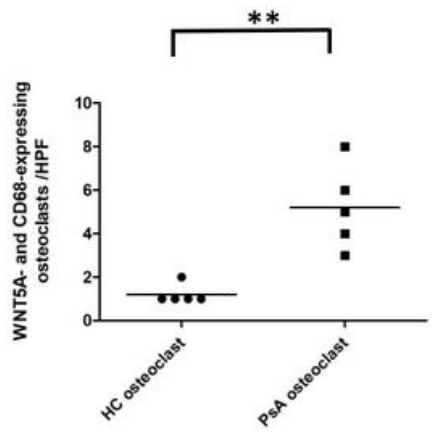

Figure 1

Increased expression levels of WNT5A in MDOC ex vivo and residential osteoclasts in the destructive joints of PsA patients. Circulatory CD14+ monocytes were obtained from PsA patients and HC through magnetic sorting. To produce MDOC, the CD14+ monocytes were treated with M-CSF for 3 days, followed by TNF- $a$ and RANKL treatment every 3 days for 9 days. (a) RNA samples from CD14+ monocytes and MDOC from healthy controls $(H C)(n=3)$ and PsA patients $(n=3)$ were analyzed to profile WNT expression using qRT-PCR. The WNT expression profile showed that WNT5A was selectively upregulated in MDOC from PsA patients compared to in those from HC. (b) The upregulation of WNT5A was reproduced in 
MDOC, according to the qRT-PCR results obtained for MDOC from HC $(n=16)$ and PsA patients $(n=32)$.

(c) The protein expression level of WNT5A in MDOC from PsA patients $(n=3)$ and HC $(n=3)$ was measured via Western blotting. (The representative data are from two PsA patients and two HC.) (d)

Immunohistochemical analyses (DAB) showed higher numbers of WNT5A (brown)-expressing and CD68 (red)-expressing osteoclasts in the destructive joints of patients with PsA $(n=5)$ than in those from patients with osteoarthritis $(n=5)$. Scale bar: $50 \mu \mathrm{m}$. * indicates $p<0.05$, ** indicates $p<0.01$, and *** indicates $p<0.001$.

a



b

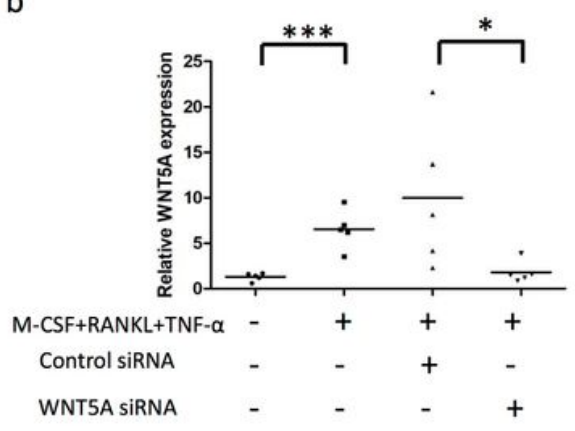

C Medium only

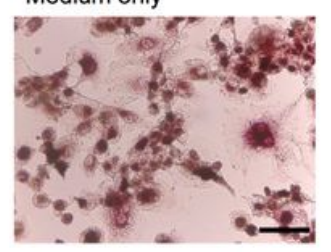

M-CSF+RANKL+TNF- $\alpha+$

Control siRNA

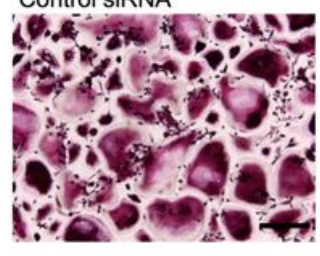

M-CSF+RANKL+TNF- $a$



M-CSF+RANKL+TNF- $\alpha+$

WNT5A SIRNA

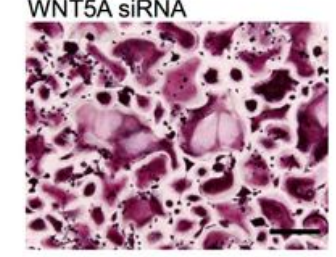

d

e

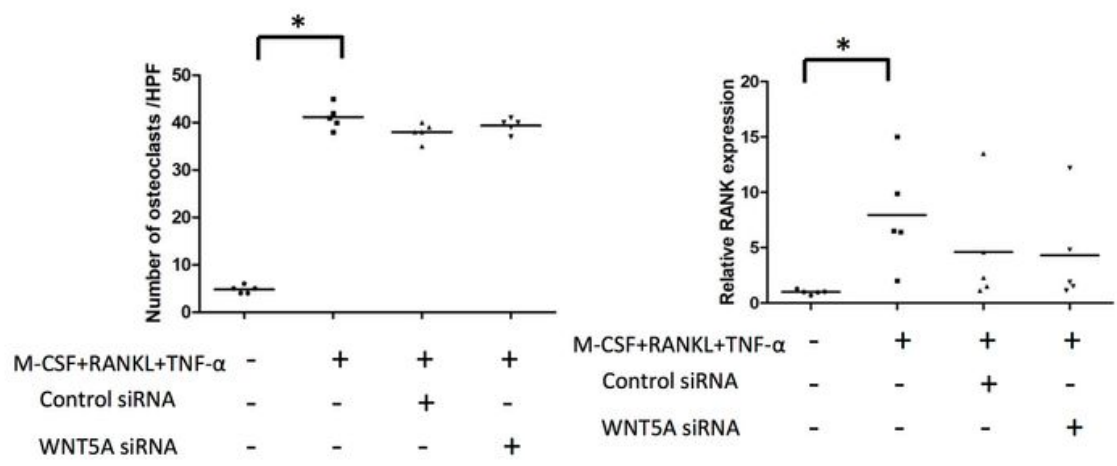

Figure 2

Osteoclastogenesis and RANK expression in MDOC is independent of WNT5A expression. The MDOC were obtained as previously described on Day 13 after treatment with RANKL and TNF-a. (a) The expression levels of WNT5A and RANK in the MDOC were measured using qRT-PCR $(n=5)$. The expression of WNT5A and RANK in CD14+ monocytes with M-CSF and TNF-a treatment was measured using qRTPCR. (b) MDOC were transfected with either control siRNA $(100 \mu \mathrm{g} / \mathrm{mL})$ or WNT5A siRNA $(100 \mu \mathrm{g} / \mathrm{mL})$ to investigate the dynamic expression level of WNT5A during MDOC differentiation using qRT-PCR. (c) MDOC transfected with control siRNA or WNT5A siRNA were identified morphologically using TRAP staining. The numbers of MDOC were determined (d) by averaging the TRAP+ cells in four high-power fields $(n=5)$. Scale bar: $50 \mu \mathrm{m}$. (e) The relative expression of RANK in MDOC transfected with control siRNA or WNT5A siRNA was measured using qRT-PCR. * ${ }^{*}$ indicates $p<0.05$ and $*$ indicates $p<0.01$. 


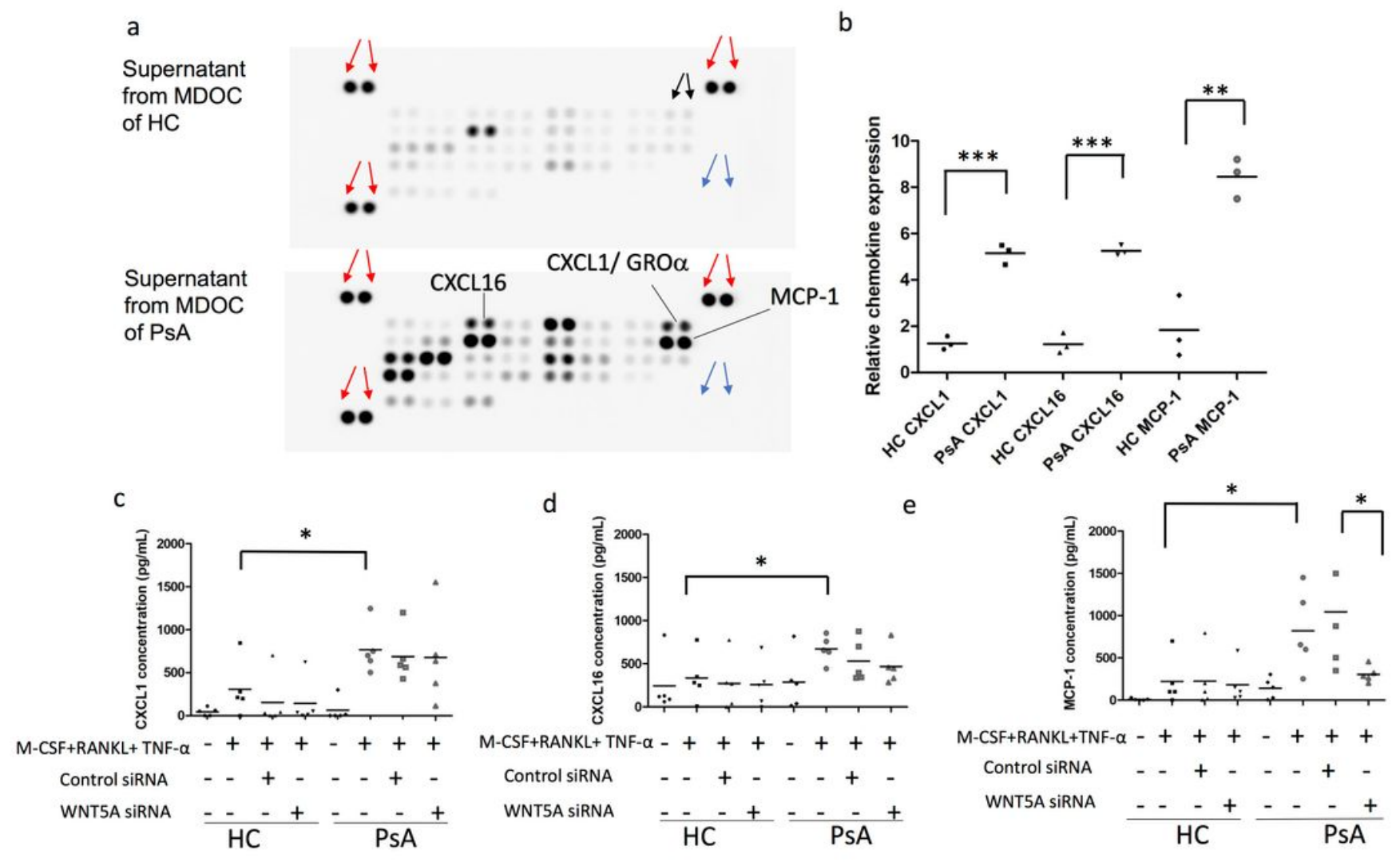

\section{Figure 3}

Selectively increased expression of MCP-1 in supernatants of MDOC from patients with PsA-dependent induction of WNT5A pathway. MDOC from PSA patients and HC were isolated as previously described. (a) On Day 13 , culture supernatants from MDOC from PsA patients $(n=3)$ and $\mathrm{HC}(n=3)$ were assayed using a human cytokine array. Positive controls were located in the upper and lower left and upper right corners (red arrows), while the negative controls were located in the right lower corner (blue arrows). The average signal intensity of each pair of cytokine spots was quantified using ImageJ and is expressed as the fold change relative to the data for HC. The dotted line denotes a fold change of 1 (black arrows). (b) CXCL1, CXCL16, and MCP-1 levels in the supernatants from MDOC of PSA patients and of HC were quantified using the data from (a) with ImageJ. (c, d, and e) MDOC from PsA patients or HC were obtained by incubation with M-CSF, RANKL, and TNF-a, with transfection of control siRNA or WNT5A siRNA. CXCL1 (c), CXCL16 (d), and MCP-1 (e) levels were determined using ELISA in culture supernatants from monocytes or MDOC with RNA interference. * indicates $p<0.05$, ** indicates $p<0.01$, and *** indicates $p<$ 0.001 . 
a
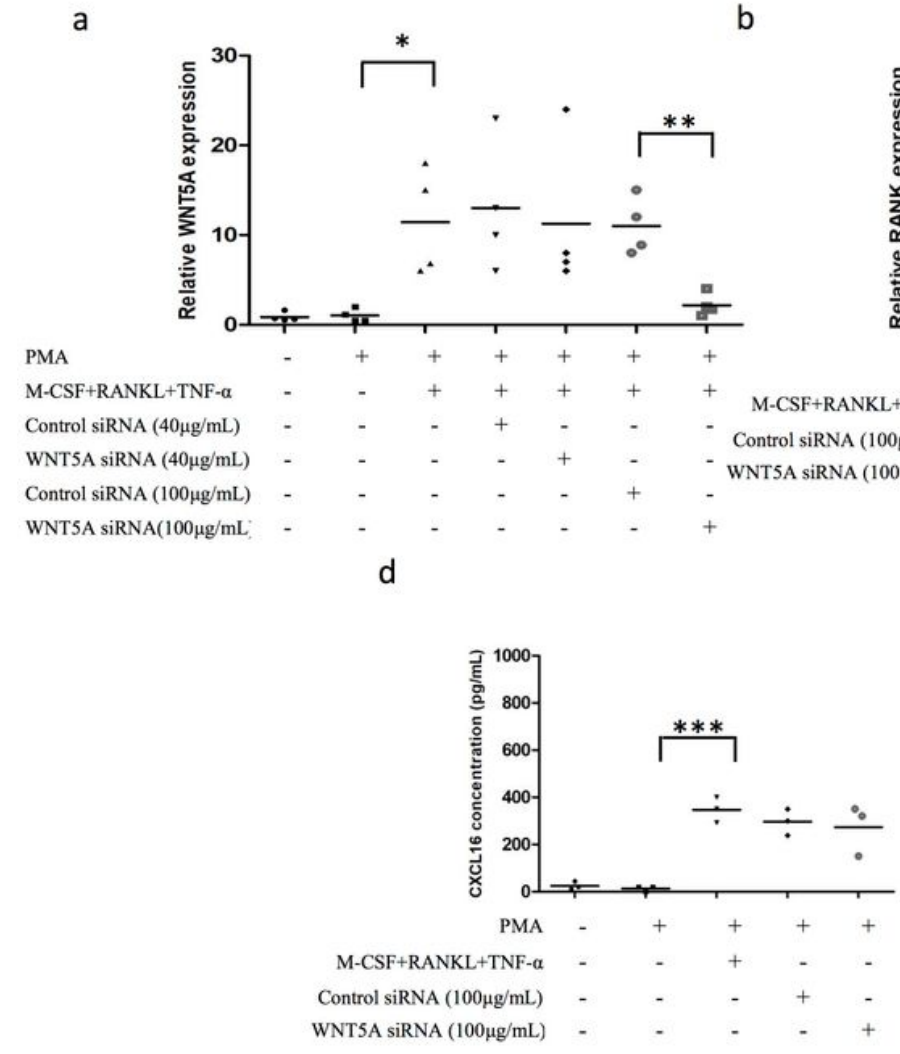

C
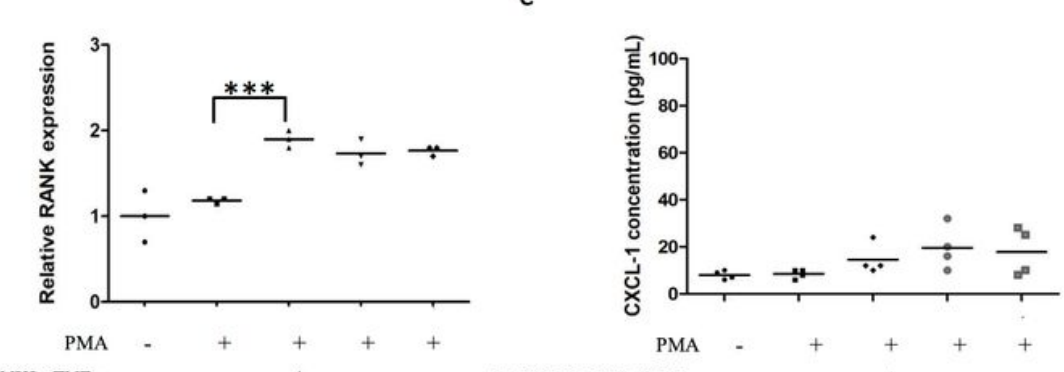

e

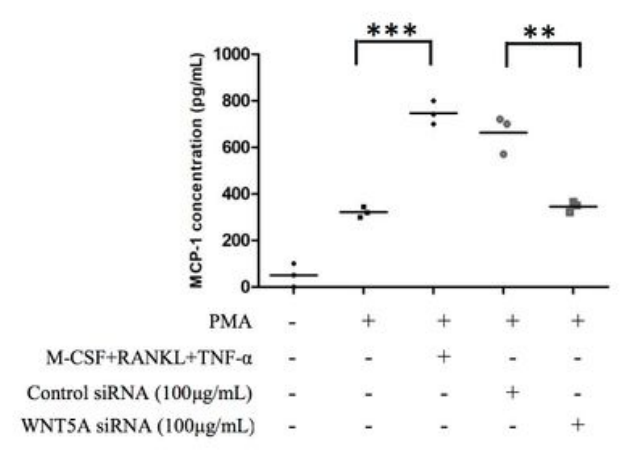

\section{Figure 4}

TNF-a induced the expression of WNT5A in THP-1-derived osteoclasts. THP-1 cells were incubated with or without PMA at $100 \mathrm{nM}$ for 24 hours. The PMA-treated THP-1 cells were then incubated with or without M-CSF $(20 \mathrm{ng} / \mathrm{mL})$, RANKL $(25 \mathrm{ng} / \mathrm{mL})$, and TNF-a $(25 \mathrm{ng} / \mathrm{mL})$ to induce osteoclasts. The M-CSF was added on Day 2, and the RANKL and TNF-a were added on Days 2 and 5. THP-1-derived osteoclasts were transfected with control siRNA or WNT5A siRNA at 40 and $100 \mu \mathrm{g} / \mathrm{mL}$ on Day 2 for 24 hours. On Day 8, the THP-1-derived osteoclasts were obtained for further measurements and experiments. (a) The expression of WNT5A in THP-1-derived osteoclasts was measured using qRT-PCR. (b) The expression of RANK in THP-1-derived osteoclasts was measured using qRT-PCR. The levels of CXCL1 (c), CXCL16 (d), and MCP-1 (e) in the supernatant from THP-1-derived osteoclasts transfected with control siRNA or WNT5A siRNA were measured using ELISA. * indicates $p<0.05$, ** indicates $p<0.01$, and *** indicates $p<$ 0.001 . 


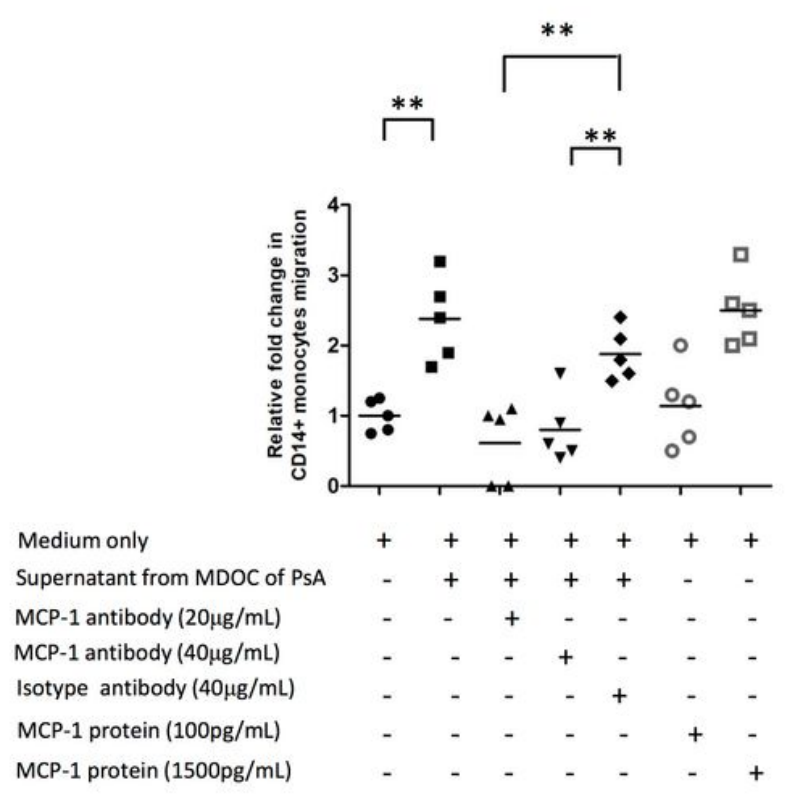

b
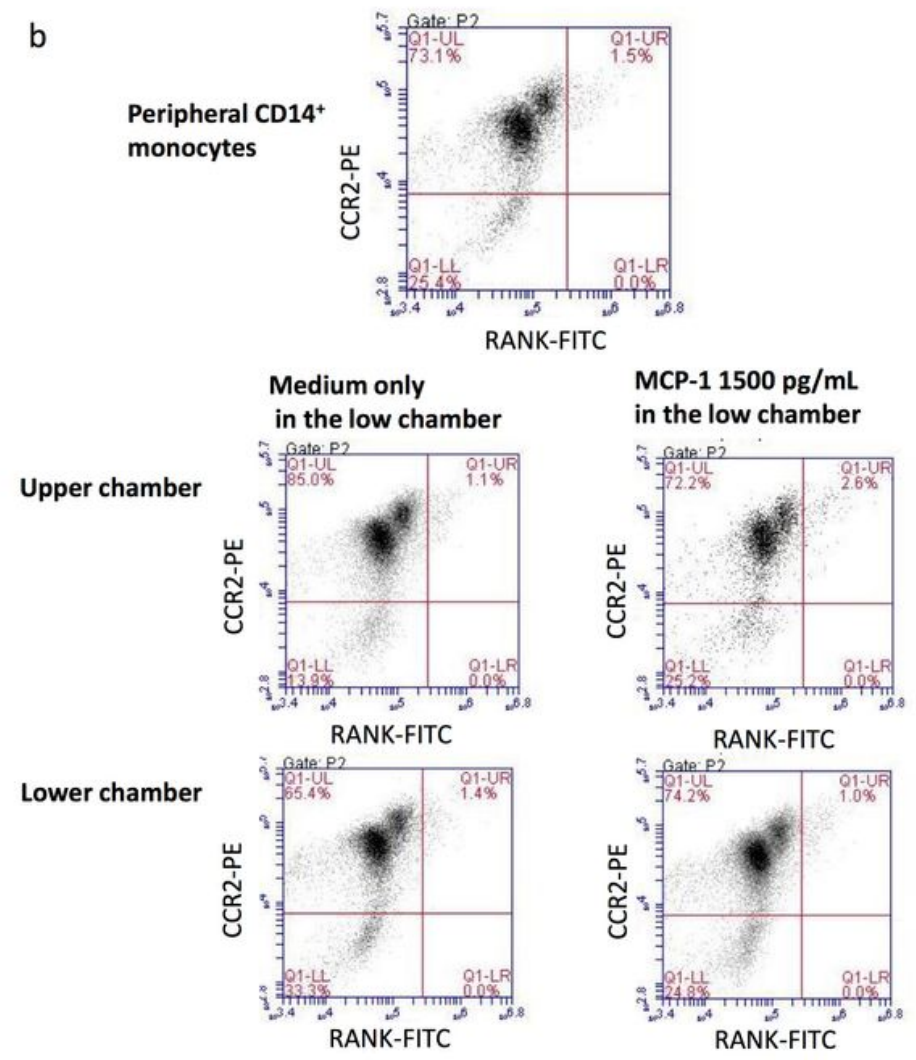

\section{Figure 5}

High recruitment of OCP induced by culture supernatant of MDOC from PsA patients could be reversed by MCP-1 antibody. MDOC were derived from CD14+ cells treated with M-CSF, RANKL, and TNF-a, from 5 PsA patients. On Day 13, the supernatants from MDOC were collected for further Transwell chemotaxis assays. (a) Supernatants were mixed with MCP-1 antibody at 20 or $40 \mathrm{ng} / \mathrm{mL}$ or corresponding isotype antibody at $40 \mathrm{ng} / \mathrm{mL}$ for the lower chamber. In other sets, culture medium supplemented with recombinant MCP-1 protein at 100 and $1500 \mathrm{pg} / \mathrm{mL}$ was placed in the lower chamber. The CD14+ monocytes from $\mathrm{HC}$ were placed above the filter (upper chamber) to allow chemotaxis for 1 hour. We measured the migration of CD14+ monocytes to the lower chamber using a Cell Counting Kit-8. (b) The numbers of CD14+ monocytes in the upper chamber and the lower chamber were determined. OCP, defined as CCR2+RANK-expressing CD14+ monocytes, were identified by multicolor flow cytometry. The secondary antibodies were conjugated with different fluorescent markers (RANK: FITC and CCR2: PE). The isotypes of individual antibodies were used as negative controls. The medium only or MCP-1 recombinant protein $(1500 \mathrm{pg} / \mathrm{mL})$ was added into the lower chamber. The percentages of CCR2+RANKexpressing CD14+ monocytes in the upper and lower chambers were measured. ${ }^{\star *} p$ indicates $<0.01$. 
a

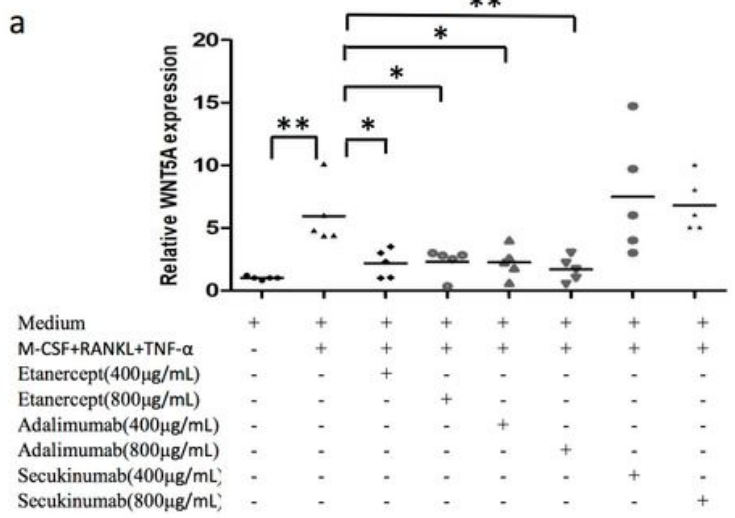

C

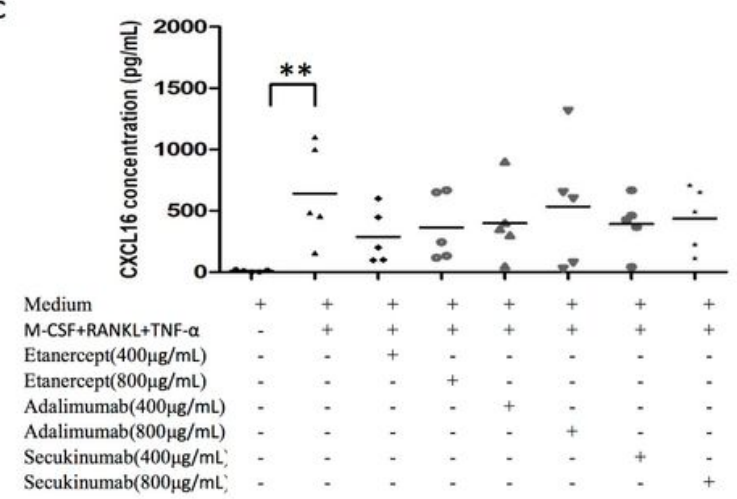

b

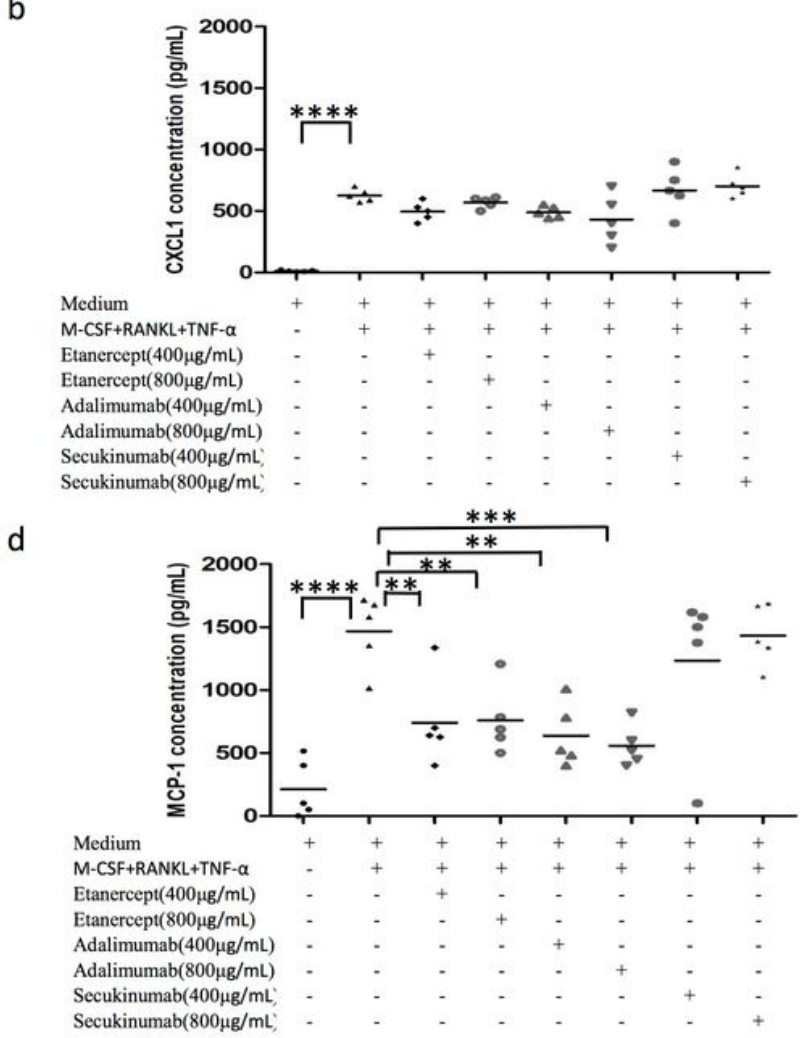

\section{Figure 6}

Anti-TNF-a agents inhibit the expression of WNT5A and production of MCP-1 from supernatants of MDOC in PsA patients. MDOC were obtained from 5 PsA patients as described previously. MDOC were treated with anti-TNF-a (adalimumab at 400 and $800 \mu \mathrm{g} / \mathrm{mL}$ or etanercept at 400 and $800 \mu \mathrm{g} / \mathrm{mL}$ ) and anti-IL-17a (secukinumab at 400 and $800 \mu \mathrm{g} / \mathrm{mL}$ ) agents on Days 3 and 9. On Day 13, the supernatants and RNA samples from MDOC were collected. (a) The expression level of WNT5A in MDOC was measured using qRT-PCR. The levels of CXCL1 (b), CXCL16 (c), and MCP-1 (d) in the supernatants were

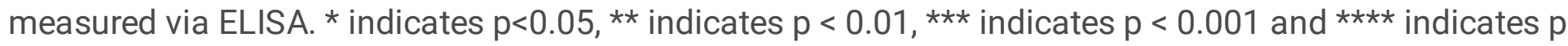
$<0.0001$.

\section{Supplementary Files}

This is a list of supplementary files associated with this preprint. Click to download.

- SUPPLEMENTARYMATERIALSANDMETHODS.docx

- SupplementaryTable1.docx

- SupplementaryTable2.docx 